\title{
Minimally invasive endoscopic resection for the treatment of sinonasal malignancy: the outcomes and risk factors for recurrence
}

This article was published in the following Dove Press journal:

Therapeutics and Clinical Risk Management

3 May 2017

Number of times this article has been viewed

\author{
Ning $\mathrm{He},{ }^{1-3}$ Xiaohong \\ Chen, ${ }^{2}$ Luo Zhang, ${ }^{2}$ Xuejun \\ Chen, ${ }^{2}$ Zhigang Huang, ${ }^{2} \mathrm{Qi}$ \\ Zhong, ${ }^{2}$ Hongzhi Ma, ${ }^{2}$ Ling \\ Feng, ${ }^{2}$ Lizhen Hou, ${ }^{2}$ Jugao \\ Fang ${ }^{1,2,4}$ \\ 'Department of Otolaryngology - \\ Head and Neck Surgery, Beijing \\ Tongren Hospital, Capital Medical \\ University, ${ }^{2} \mathrm{Key}$ Laboratory of \\ Otorhinolaryngology, Head and Neck \\ Surgery, Ministry of Education, Beijing \\ Institute of Otorhinolaryngology, \\ Beijing, ${ }^{3}$ Department of \\ Otolaryngology \& Head and Neck \\ Oncology, The People's Hospital \\ of Guangxi Zhuang Autonomous \\ Region, Nanning, Guangxi Zhuang \\ Autonomous Region, ${ }^{4}$ Beijing Key \\ Laboratory of Head and Neck \\ Molecular Diagnostic Pathology, \\ Beijing, People's Republic of China
}

Correspondence: Jugao Fang Department of Otolaryngology - Head and Neck Surgery, Beijing Tongren Hospital, Capital Medical University, I Dongjiaominxiang, Dongcheng, Beijing 100703, People's Republic of China $\mathrm{Tel} / \mathrm{fax}+861058269512$

Email fangjugao@।63.com

\section{Xiaohong Chen}

Key Laboratory of Otorhinolaryngology, Head and Neck Surgery, Ministry of Education, Beijing Institute of Otorhinolaryngology, I Dongjiaominxiang, Dongcheng, Beijing 100005, People's Republic of China $\mathrm{Tel} / \mathrm{fax}+8610582695 / 2$ Email trchenxh@।63.com
Purpose: The role of minimally invasive endoscopic resection (MIER) in the treatment of sinonasal malignancy is controversial. Herein, we performed a retrospective review of a large case series of sinonasal malignancy patients treated with MIER aimed at evaluating the outcomes and identifying the risk factors for recurrence.

Methods: Patients with sinonasal malignancy who underwent MIER from March 2000 to May 2015 were enrolled, and their clinical data were collected. The clinical outcomes were evaluated by determining the 5-year overall survival (OS) and disease-free survival (DFS). The predictive factors for survival and potential independent risk factors for recurrence were explored.

Results: A total of 120 patients were enrolled, including 62 males and 58 females. The mean follow-up period was 51.4 (95\% confidence interval: 44.0-59.1) months. The most frequent histological type was mucosal malignant melanoma. The positive margin rate was $19.2 \%(23 / 120)$. Seventy-one patients had the safety anatomic plane (SAP). Age $\geq 50$ years, nodal metastasis, and not having the SAP were found to be predictive factors for survival, and absence of SAP was found to be an independent risk factor for recurrence.

Conclusion: Our study indicated that MIER is an effective and safe surgical procedure in appropriately selected patients. Tumor resection with a safety anatomic boundary is likely to lead to improved survival and decreased recurrence. However, a larger sample and longterm prospective observation are still required to establish the role of MIER in treatment of sinonasal malignancy.

Keywords: minimally invasive endoscopic resection, sinonasal skull base, malignancy, outcome, recurrence

\section{Introduction}

Endonasal endoscopic surgery, also named minimally invasive endoscopic resection (MIER), has been used to manage sinonasal malignancy (SNM) since the 1990s. ${ }^{1-3}$ Over the past 2 decades, although MIER has become an increasingly practiced procedure, the role of endoscopic surgery in the treatment of SNM remains undetermined. ${ }^{4,5}$ Clinical studies have reported that MIER offers an equivalent benefit in terms of patients' survival compared to anterior craniofacial resection (ACFR), improves patients' quality of life, and decreases postoperative morbidity in carefully selected patients., ${ }^{3,5-8}$ However, the late presentation of SNM and its juxtaposition to important anatomical structures such as eyes and brain pose a challenge to surgical management: what should be the proper extent of resection? There is little discussion on this topic in existing literature. Due to the low incidence of SNM and insufficient evidence, a widely accepted 
standard practice is yet to be established. In our study, we analyzed the clinical outcomes of 120 SNM patients who underwent MIER and aimed to identify the predictive factors for survival and the risk factors for recurrence. The extent to which resection can bring about favorable outcomes was also explored.

\section{Materials and methods Study design}

This study was reviewed and approved by the ethics committee of Beijing Tongren Hospital. All the patients recruited in this study provided written informed consent. A database search in the Department of Head and Neck Surgery, Beijing Tongren Hospital, was performed to identify patients from March 2000 to May 2015 who had been pathologically diagnosed to have SNM, undergone radical resection with MIER, and had a follow-up $\geq 6$ months after the surgery. Medical records of eligible patients were reviewed to gather information on demographics, clinical diagnosis, and treatment, including disease stage, surgical management, complication, and adjuvant therapy. Information from routine postoperative follow-up sessions was also collected, including the length of follow-up and disease status at the latest follow-up. All patients provided informed consent before treatment and were systematically evaluated by endoscopy, high-resolution computed tomography (CT), sinonasal magnetic resonance imaging (MRI) scans, and whole-body positron emission tomography (PET)-CT scan. A multidisciplinary team consisting of a pathologist, radiologist, radiation physician, chemotherapy physician, and surgeons assessed the disease staging and determined the treatment plan, including surgical procedure, reconstruction, and adjuvant therapy strategy before the surgery. Recurrence and metastasis were verified using imaging and pathological examination.

\section{Surgical procedure}

The extent of surgical removal was determined according to the tumor size, and the surgical margin was controlled by using the frozen section approach. The safety anatomic boundary (SAB) was defined as a layer of normal tissues, such as bone plate, periosteum, fascia, or dura mater, between the tumor and important structures and organs that may function as a barrier limiting the growth of the tumor. We defined the plane that was exposed after resection of the tumor and SAB as the safety anatomic plane (SAP). An adequate resection included both resecting a tumor and $\mathrm{SAB}$ as well as obtaining a SAP. For example, when the tumor involved inferior ethmoidal cells, in addition to the tumor and inferior cells, the normal upper ethmoidal cells were also removed. Similarly, when the skull base bone was infiltrated, in addition to the tumor and skull base bone, the adjacent normal dura was also resected; when the orbit bone was involved, in addition to the tumor and orbit bone, the normal orbital periosteum was also removed. After resection of tumor and the SAB, the SAP was obtained. Several methods were utilized to reconstruct the defective skull base, including the use of nasoseptal flap, fascia flap, and synthetic materials, if necessary.

\section{Adjuvant therapy}

Adjuvant therapies included radiotherapy, chemotherapy, and concurrent chemoradiotherapy. According to the assessment by the team, treatment with adjuvant therapy was performed pre- or/and postoperation. Radiotherapy was completed using three-dimensional conformal radiotherapy or simultaneousboost intensity-modulated radiotherapy. Radiation doses ranged from 60 to 77.88 Gy. Preoperative chemotherapy or chemoradiotherapy was implemented instead when the tumors were too large to be radically resected and the SAP obtained, when they were adjacent to important organs or structures such that the surgery may have led to impaired functions, or/and when the patients were reluctant or unsuitable for an open approach. After two to three cycles of chemotherapy with or without radiotherapy, the tumors were assessed again. If they had shrunk, the tumors were considered for complete resection; the SAPs could be obtained after a reassessment, and MIER then followed. Otherwise, an open approach was adopted.

\section{Statistical methods}

The Kaplan-Meier method and log-rank test were used for survival analysis. Predictive factors regarding survival and recurrence were analyzed using Cox proportional hazard regression. Statistical analysis was performed using the SPSS 20.0 statistical package (IBM Corp, Armonk, NY, USA). A $P$-value $<0.05$ was considered statistically significant.

\section{Results}

\section{Clinical characteristics}

A total of 120 patients were collected in our study, including 62 males and 58 females, with a male-to-female ratio of 1.07:1. The mean age was 48.1 (95\% confidence interval [CI]: 45.0-51.0) years. The mean postoperation follow-up was for 51.4 (95\% CI: 44.0-59.1) months. Three patients were lost during follow-up after discharge from the hospital. 
Based on the tumor-node-metastasis (TNM) staging system of the American Joint Committee on Cancer, ${ }^{9}$ tumors were staged as follows: T1: $25.8 \%(31 / 120), \mathrm{T} 2: 21.7 \%$ (26/120), T3: $19.2 \%$ (23/120), and T4: 33.3\% (40/120); N0: $93.3 \%(112 / 120), \mathrm{N} 1: 5.8 \%(7 / 120)$ and N3: $0.8 \%$ $(1 / 120)$. The tumor locations were as follows: nasal cavity $96.7 \%(116 / 120)$, ethmoid sinus $85 \%(102 / 120)$, maxillary sinus $77.5 \%$ (93/120), sphenoid sinus $31.7 \%$ (38/120), skull base $12.5 \%$ (15/120), lamina papyracea $4.17 \%(5 / 120)$, intraorbital region $9.2 \%(11 / 120)$, dura mater $3.3 \%(4 / 120)$, pterygopalatine fossa $8.3 \%$ (10/120), infratemporal fossa $9.2 \%(11 / 120)$, and brain parenchyma 2.5\% (3/120). Among all patients, 103 cases were determined to be appropriate for MIER after first assessment, and the other 17 cases were selected for MIER at the second assessment after preoperative adjuvant therapy. Of these 17 cases that underwent preoperative therapy, eleven had intraorbital tumors, one had a tumor in the dura mater, two had tumors in the pterygopalatine fossa, and three had tumors in the infratemporal fossa.

The most frequent histology types were mucosal malignant melanoma $(22.5 \%, 27 / 120)$, sarcoma $(15.8 \%, 19 / 120)$, olfactory neuroblastoma (ONB) $(15.0 \%, 18 / 120)$, and squamous cell carcinoma (SCC) $(13.3 \%, 16 / 120)$. Adenoid cystic carcinoma and adenocarcinoma each accounted for $8.3 \%(10 / 120)$. Other tumor types were plasma cell carcinoma $(4.2 \%, 5 / 120)$, carcinosarcoma $(3.3 \%, 4 / 120)$, acinic cell carcinoma $(2.5 \%, 3 / 120)$, neuroendocrine carcinoma $(1.7 \%, 2 / 120)$, solitary fibrous tumor $(1.7 \%, 2 / 120)$, lymphoepithelioma $(0.8 \%, 1 / 120)$, clear cell carcinoma $(0.8 \%, 1 / 120)$, mucoepidermoid carcinoma $(0.8 \%, 1 / 120)$, and myoepithelial carcinoma $(0.8 \%, 1 / 120)$.
Table I Surgical margins in the without-SAP group

\begin{tabular}{lllllll}
\hline Margins & SS, $\mathbf{n}$ & $\mathbf{P F}, \mathbf{n}$ & IF, $\mathbf{n}$ & $\mathbf{D M}, \mathbf{n}$ & $\mathbf{B P}, \mathbf{n}$ & Total, $\mathbf{N}$ \\
\hline Positive & II & 4 & 5 & 2 & $\mathrm{I}$ & 23 \\
Negative & 16 & 3 & 3 & 2 & 2 & 26 \\
Total & 27 & 7 & 8 & 4 & 3 & 49 \\
\hline
\end{tabular}

Abbreviations: SAP, safety anatomic plane; SS, sphenoid sinus; PF, pterygopalatine fossa; IF, infratemporal fossa; DM, dura mater; BP, brain parenchyma.

\section{Surgery}

All 120 patients underwent radical resection, and five of them also underwent neck dissection simultaneously. In total, 71 patients obtained a SAP during the operation, but the others $(n=39)$ did not. All the tumors in the without-SAP group were in advanced stages, involving sites scattered in the sphenoid sinus, pterygopalatine fossa, infratemporal fossa, dura mater, and brain parenchyma. Twenty-three patients in the without SAP group had positive margins (Table 1). After resection, 13 received skull base reconstruction. Intraoperative successive endoscopic pictures of an esthesioneuroblastoma case are shown in Figure 1.

Three patients developed postoperative complications $(2.5 \%, 3 / 120)$, including one case $(0.8 \%, 1 / 120)$ of nasal bleeding (which was cured by nasal packing) and two patients who underwent skull base reconstruction developed cerebrospinal fluid (CSF) leakage (1.7\%, 2/120), which was later cured by conservative therapy. All 13 patients were aged $>70$ years $(10.8 \%, 13 / 120)$ and had no surgery-related complication or mortality.

\section{Adjuvant therapy}

Overall, 27 (22.5\%) patients underwent MIER alone, $17(14.2 \%)$ patients received preoperative adjuvant therapy
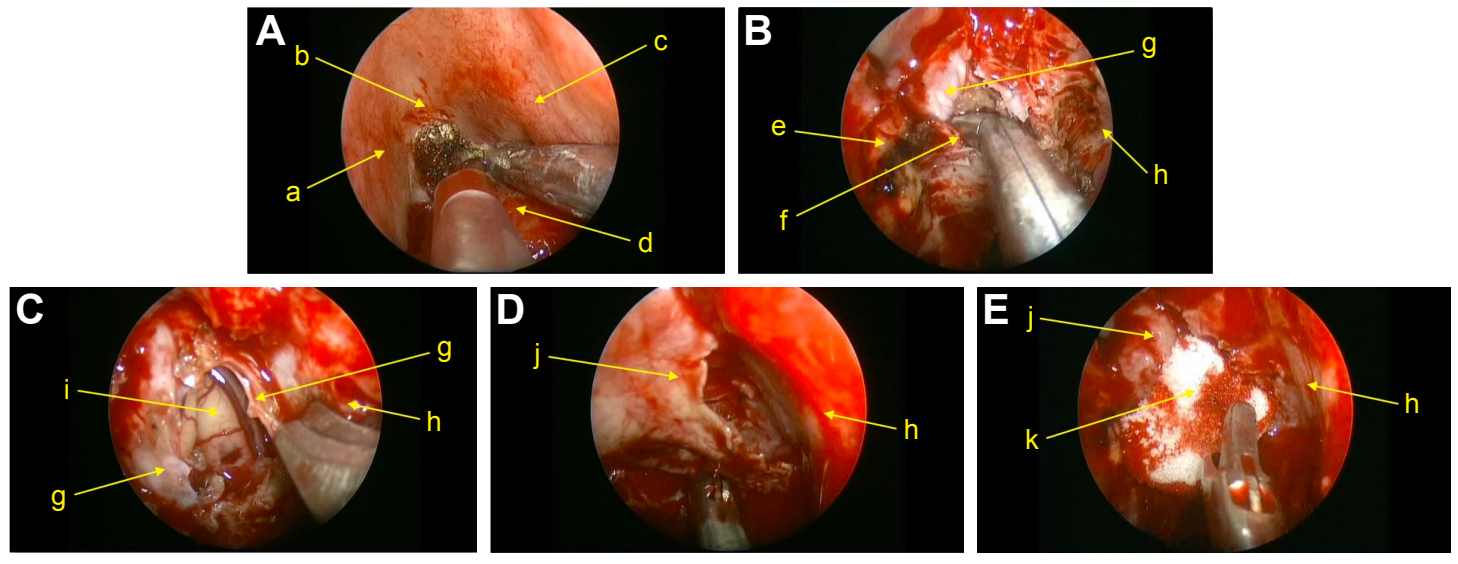

Figure I Intraoperative successive endoscopic images of an esthesioneuroblastoma case.

Notes: (A) Resecting the base of tumor, (B) resecting the cribriform plate, (C) resecting the dura mater, (D) reconstructing the skull base defect with nasoseptal flap, and (E) fixing the nasoseptal flap with hemostasis material. a: middle turbinate of right side; b: olfactory cleft of right side; c: nasal septum; d: tumor; e: lateral wall of the right ethmoid sinus; f: cribriform plate; g: dura mater; h: lateral wall of the left ethmoid sinus; i: brain parenchyma; j: nasoseptal flap; k: hemostasis material. 
Table 2 Histological type distribution in the preoperative therapy group

\begin{tabular}{llll}
\hline Histological type & Pre-C, $\mathbf{n}$ & Pre-R, $\mathbf{n}$ & Pre-CR, \\
\hline Squamous cell carcinoma & 0 & 2 & 0 \\
Olfactory neuroblastoma & 0 & 2 & 0 \\
Carcinosarcoma & 0 & 1 & 0 \\
Sarcoma & 9 & 0 & 2 \\
Adenocarcinoma & 1 & 0 & 0 \\
Total & 10 & 5 & 2 \\
\hline
\end{tabular}

Abbreviations: Pre-C, preoperative chemotherapy; Pre-R, preoperative radiotherapy; Pre-CR, preoperative chemoradiotherapy; n: number.

followed by MIER (Table 2) and postoperative therapy, and 76 (63.3\%) patients underwent MIER followed by postoperative therapy.

\section{Survival, recurrence, and metastases}

The crude 5-year overall survival (5-OS) (Figure 2A) and 5-year disease-free survival (5-DFS) (Figure 2B) were 68.3\% and $66.2 \%$, respectively. In particular, 18 (15.4\%) cases recurred locally during a median follow-up of 46 months, four $(3.4 \%)$ developed cervical node metastasis during a median follow-up of 38 months, and 17 (14.5\%) developed distant metastases during a median follow-up of 52 months, including four lung metastases, four liver metastases, two bone metastases, two brain metastases, and five multiorgan metastases. The 5-OS and 5-DFS varied by individual histological type (Table 3 ) and adjuvant treatments (Table 4). Patients with ONB had the longest survival time, followed by those with SCC, mucosal malignant melanoma, and sarcoma. Patients with SAP also had better survival outcomes than did the patients without SAP (5-OS: $85.1 \%$ vs $41.2 \%$, Figure 3 A;
5-DFS: $83.3 \%$ vs 38.8\%, Figure 3B). Representative MRIs scans of a patient with ONB (Figure 4A-D) and another patient with right ethmoid rhabdomyosarcoma (Figure 5A-C) are shown. The images were captured before and after surgery in combination with radiotherapy and chemoradiotherapy, respectively, as indicated.

\section{Predictive factors for OS, DFS, and recurrence}

A Cox regression analysis showed that the independent predictive factors for OS were age $\geq 50$ years (hazard ratio $[\mathrm{HR}]=4.32,95 \% \mathrm{CI}=1.64-11.37, P=0.01)$, cervical nodal metastasis $(\mathrm{HR}=18.53,95 \% \mathrm{CI}=3.24-105.97, P=0.01)$, positive margin $(\mathrm{HR}=4.32,95 \% \mathrm{CI}=1.64-11.37, P=0.01)$, histology $(P=0.01)$, and without SAP (HR $=7.93,95 \%$ $\mathrm{CI}=2.18-28.82, P=0.01)$; for DFS, they were age $\geq 50$ years $(\mathrm{HR}=2.59,95 \% \mathrm{CI}=1.64-6.02, P=0.03)$, cervical nodal metastasis $(\mathrm{HR}=8.61, \mathrm{CI}=1.70-43.60, P=0.01)$, histology $(P=0.04)$, and absence of SAP $(\mathrm{HR}=7.71,95 \%$ $\mathrm{CI}=2.32-25.63, P=0.01)$; and for recurrence, they were absence of SAP only $(\mathrm{HR}=7.12,95 \% \mathrm{CI}=1.43-35.56$, $P=0.01)$. Different histological types have diverse survival rates $(P=0.01)$ : melanoma produced a higher risk of mortality than ONB ( $\mathrm{HR}=16.03,95 \% \mathrm{CI}=1.54-166.4$, $P=0.02)$ and $\mathrm{SCC}(\mathrm{HR}=11.97,95 \% \mathrm{CI}=1.32-108.71$, $P=0.03$ ) when focusing on $\mathrm{OS}$, with similar results when focusing on DFS $(\mathrm{HR}=12.86,95 \% \mathrm{CI}=1.33-124.21$, $P=0.03 ; \mathrm{HR}=12.31,95 \% \mathrm{CI}=1.45-104.69, P=0.02$, respectively); there was no statistical significance when melanoma was compared to sarcoma $(P=0.17)$ and the other cancer groups $(P=0.28)$ (Table 5$)$.
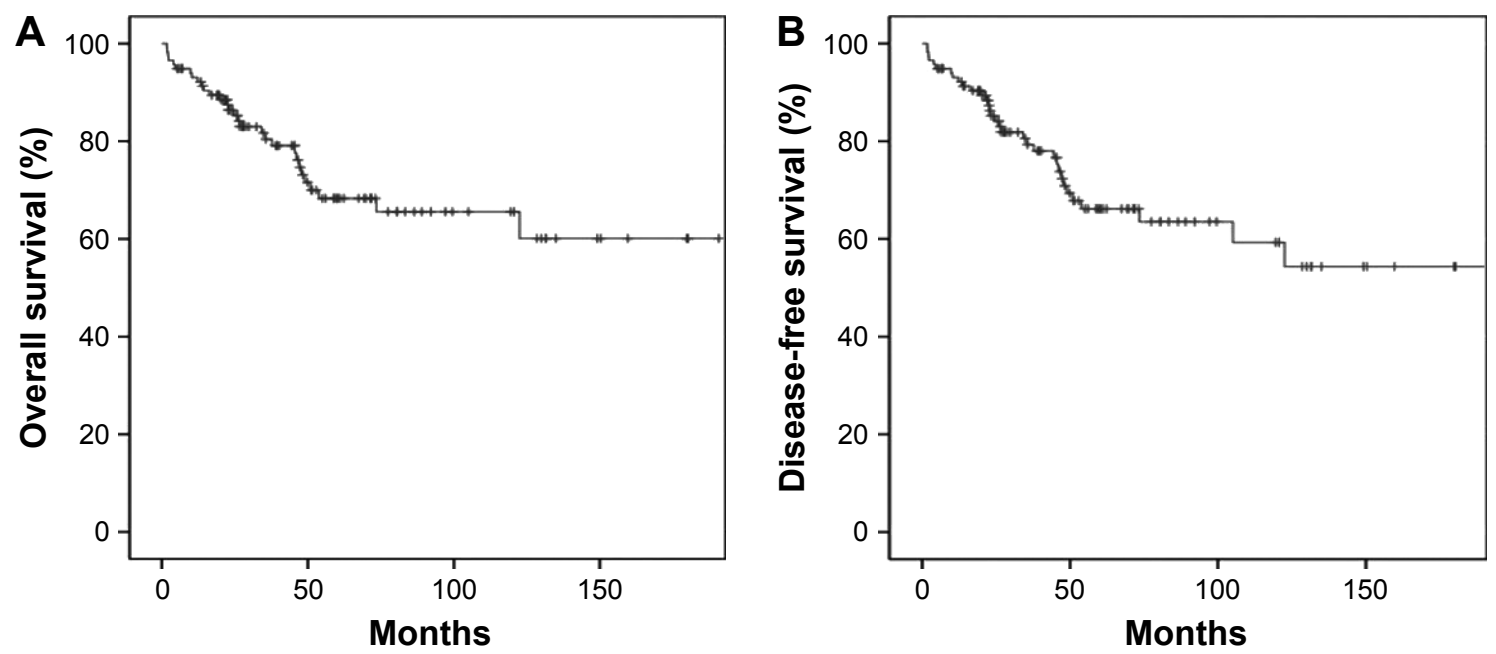

Figure 2 Clinical outcomes in patients with sinonasal malignancy who received MIER. Notes: (A) Overall survival. (B) Disease-free survival.

Abbreviation: MIER, minimally invasive endoscopic resection. 
Table 3 Clinical outcomes by tumor histology

\begin{tabular}{lllll}
\hline Histology & N & $\begin{array}{l}\text { 5-year } \\
\text { OS, \% }\end{array}$ & $\begin{array}{l}\text { 5-year } \\
\text { DFS, \% }\end{array}$ & Mean OS \pm SE (95\% CI) \\
\hline Whole cohort & 117 & 68.3 & 66.2 & $132.38 \pm 9.06($ II4.62-I50.14) \\
MMM & 27 & 37 & 37 & $70.96 \pm 12.78(45.91-96.01)$ \\
ONB & 18 & 94.4 & 94.4 & $180.93 \pm 9.56(162.19-199.67)$ \\
SCC & 16 & 54.1 & 54.1 & $85.05 \pm 14.14(57.33-112.76)$ \\
Sarcoma & 17 & 61.4 & 50.1 & $63.6 I \pm 8.31(43.33-79.89)$ \\
Others & 39 & 77.6 & 74.5 & $103.48 \pm 12.81(78.37-128.59)$ \\
\hline
\end{tabular}

Abbreviations: $N$, number; OS, overall survival; DFS, disease-free survival; $S E$, standard error; $\mathrm{Cl}$, confidence interval; MMM, mucosal malignant melanoma; ONB, olfactory neuroblastoma; SCC, squamous cell carcinoma.

\section{Discussion}

Since the 1960s, ACFR has been the standard treatment for paranasal sinus tumors, particularly when the tumor invades the anterior skull base. ${ }^{3}$ The advantages of this approach are its wide exposure and flexibility to perform bloc resection with adequate margins. However, it also results in major complications. According to the literature, the 5-OS, postoperative complication rate, and postoperative mortality rate were $54 \%, 33 \%$, and $4 \%$, respectively. ${ }^{10} \mathrm{With}$ the development of the endonasal endoscopic technique, MIER has exhibited its superiority as it can maximally avoid skull handling and retraction of brain tissue and therefore results in better quality of life and less unnecessary intercurrent injuries, which has been expected to provide an alternative modality for SNM management with equivalent outcomes and decreased complication rate as compared to ACFR ${ }^{8,11-15}$ However, due to the small sample size, mixed histological types, and short postoperative follow-up, the results of previous MIER studies have not been widely accepted. ${ }^{2,6}$ In our study, not only the crude but also the histology-specific 5-OS and 5-DFS were higher when compared to those of ACFR reported in previous studies. ${ }^{10}$ Our data also showed a lower rate of CSF leak, which is the most common complication of ACFR, ${ }^{16}$ and a decreased overall rate of postoperative complication and mortality. The results thus provide the evidence for the fact that MIER is an effective and safer alternative to open surgery in carefully selected patients, which is attributable to developments in terms of endoscopic instruments and hemostasis materials, improvement of skull base anatomic knowledge, and the accumulating experiences of a surgical team. Magnified and high-definition vision enables the preservation of important adjacent regions during the tumor resection process. Additionally, improved reconstruction technology for skull base defects decreases the CSF leakage rate dramatically. Moreover, the multidisciplinary management optimizes the therapeutic strategies, which renders the operation safer and more effective.

Nevertheless, managing SNM by using MIER remains debatable. Endonasal endoscopic surgery is controversial because it violates the treatment principle of oncology: complete en bloc excision of the tumor with adequate margins. ${ }^{4,17}$ Fortunately, the piecemeal resection of MIER has been shown to not undermine the patients' survival, ${ }^{18}$ and what remains to be determined is the extent of surgical resection to achieve the radical removal in the skull base when encompassed by important structures while preserving the function of important structures at the same time.

In the multivariate analysis of this study, age $\geq 50$ years, cervical nodal metastasis, and absence of SAP are the common independent predictive factors when focusing on 5-OS and 5-DFS, and absence of SAP is also the risk factor for recurrence and contributes to both decreased survival and increased recurrence. Therefore, obtaining a SAP is important to the surgical procedure; we believe that after resecting both the tumor and an adjacent layer of normal tissue, an oncologic safe plane should be obtainable. In other words, a radical resection should resect both the tumor and $\mathrm{SAB}$, thereby obtaining a SAP, which is supposed to increase the survival and decrease the recurrence. Castelnuovo et $\mathrm{al}^{19}$ thought that the concept of the "oncologic plane" is very important in the management of malignancies, and the plane

Table 4 Clinical outcomes by pre- and postoperative therapy

\begin{tabular}{|c|c|c|c|c|}
\hline $\begin{array}{l}\text { Therapeutic } \\
\text { modality }\end{array}$ & $\mathbf{N}$ & 5-OS, \% & 5-DFS, \% & $\begin{array}{l}\text { Mean } \pm \text { standard } \\
\text { deviation }(95 \% \mathrm{Cl})\end{array}$ \\
\hline \multicolumn{5}{|l|}{ Preoperative therapy } \\
\hline Yes & 15 & 45.8 & 45.8 & $49.08 \pm 9.61(30.64-67.52)$ \\
\hline No & 102 & 71.1 & 68.6 & 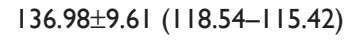 \\
\hline \multicolumn{5}{|l|}{ Postoperative therapy } \\
\hline No & 27 & 57.4 & 54.5 & $91.25 \pm 12.23(67.29-\mid 15.21)$ \\
\hline Chemotherapy & 13 & 50.1 & 50.1 & $53.38 \pm 11.13(36.56-80.20)$ \\
\hline Radiotherapy & 45 & 89 & 88.6 & 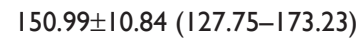 \\
\hline Chemoradiotherapy & 32 & 47.5 & 43.2 & $107.04 \pm \mid 4.80(68.23-\mid 45.86)$ \\
\hline
\end{tabular}

Abbreviations: 5-OS, 5-year overall survival; 5-DFS, 5-year disease-free survival; $\mathrm{Cl}$, confidence interval. 

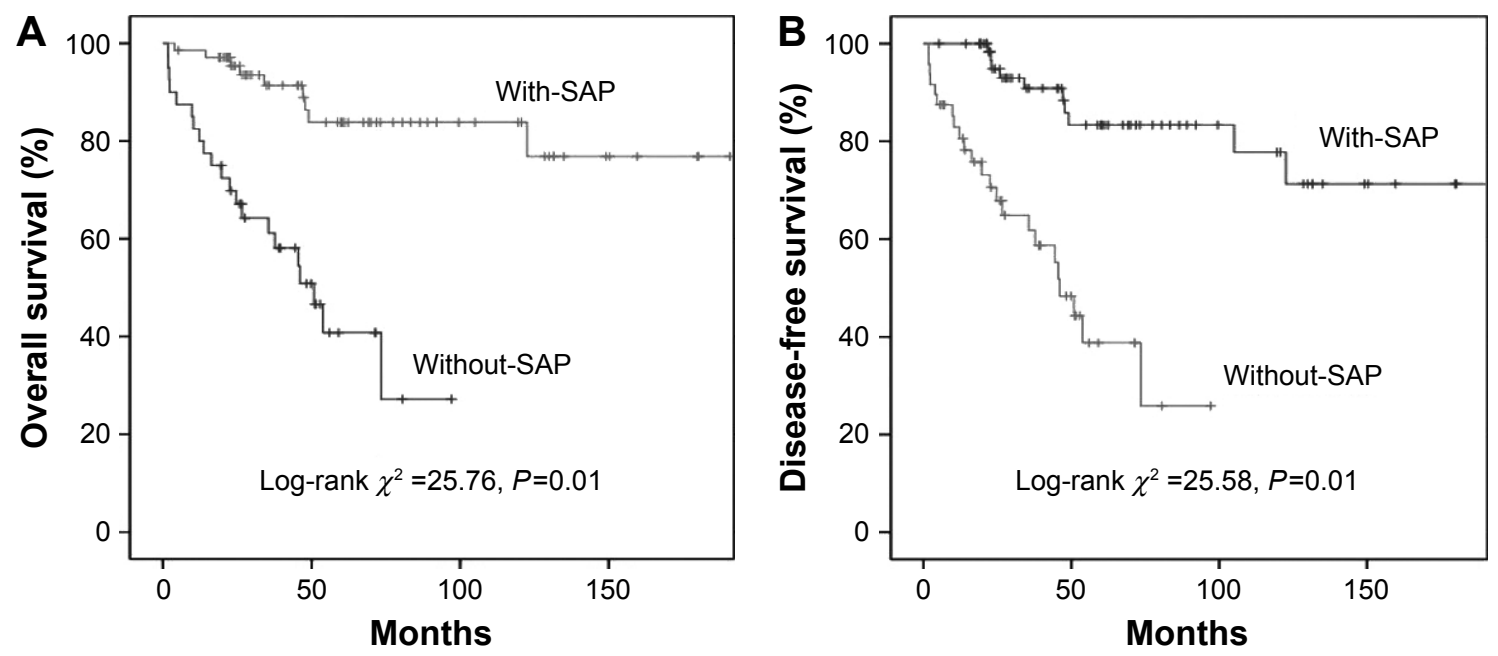

Figure 3 Clinical outcomes in the with-SAP and without-SAP groups.

Notes: (A) Overall survival. (B) Disease-free survival.

Abbreviation: SAP, safety anatomic plane.
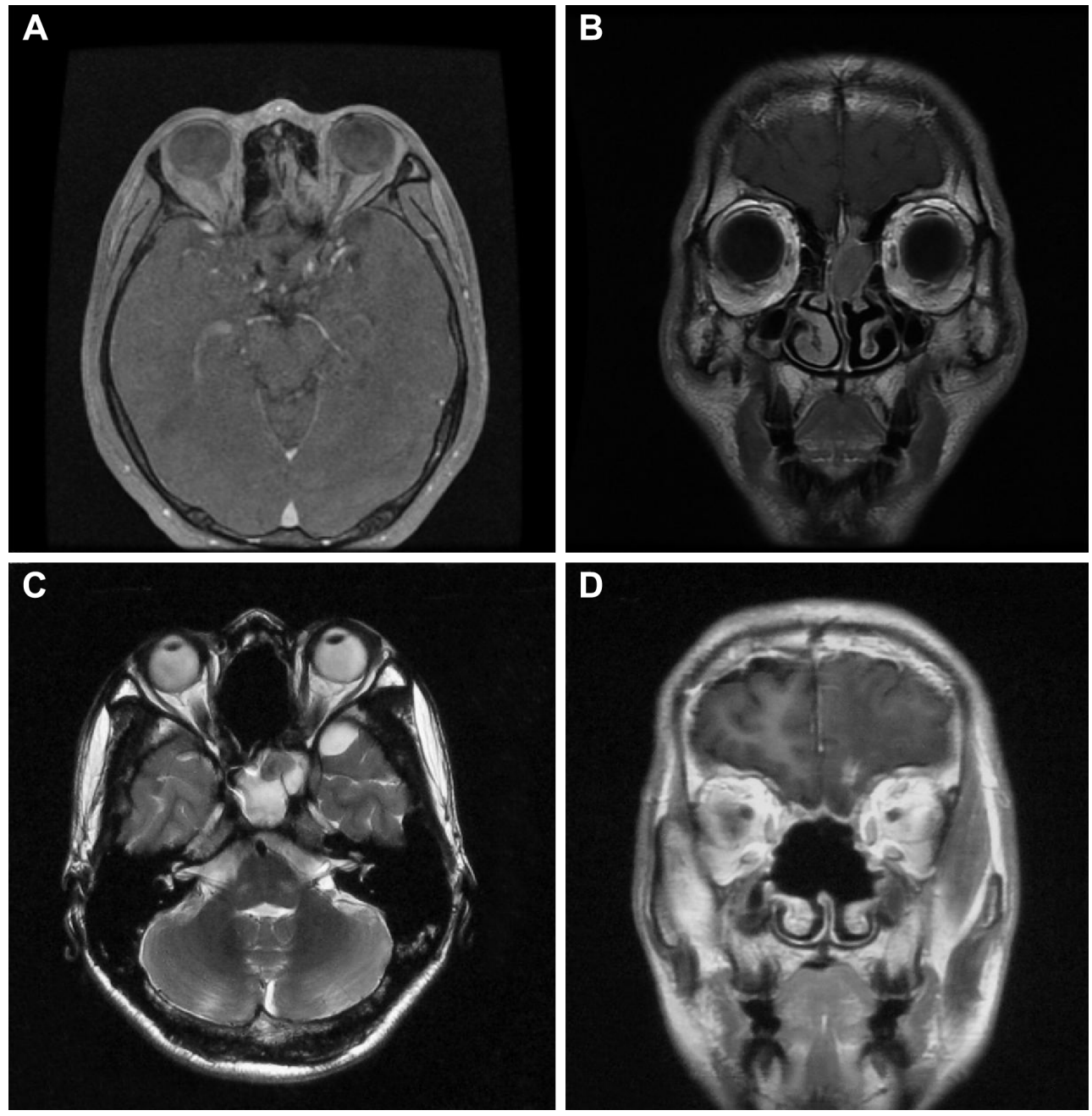

Figure 4 Representative MRI scans of a patient with olfactory neuroblastoma.

Notes: Preoperative (A) axial and (B) coronal TI-weighted MRI scans of olfactory neuroblastoma showing the tumor involving the left nasal cavity, ethmoid sinus, nasal septum, cribriform plate, and dura. The nasal septum, bilateral ethmoid sinus, cribriform plate, crista galli, olfactory bulb, and corresponding dura were resected, and a fascia flap and synthetic materials were used for reconstruction of the skull base intraoperatively. (C) Axial T2-weighted MRI and (D) coronal TI-weighted MRI scans captured 3 years after surgery and postoperative radiotherapy showing no tumor recurrence.

Abbreviation: MRI, magnetic resonance imaging. 

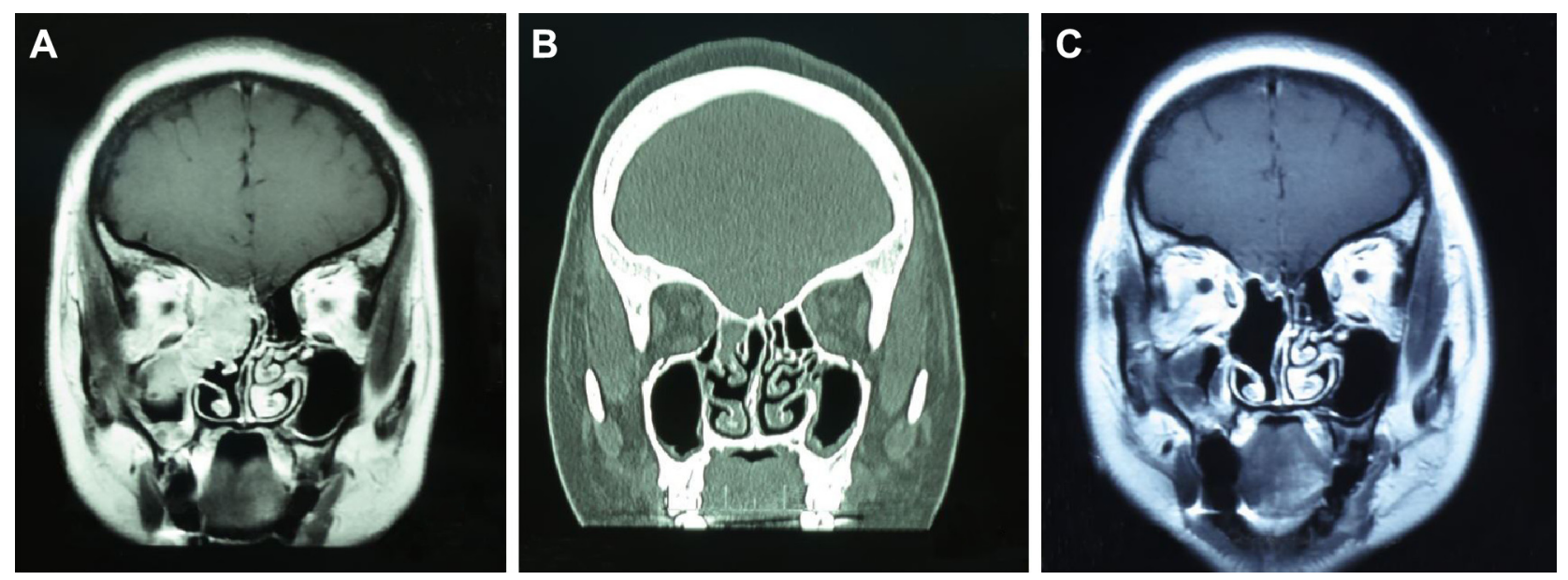

Figure 5 Representative imaging scans of a patient with right ethmoid sinus rhabdomyosarcoma.

Notes: Coronal TI-weighted MRI and CT scans of tumor extent including (A) primary state, (B) after two cycles of preoperative chemotherapy, and (C) I year after surgery followed by postoperative chemoradiotherapy. Minimally invasive endoscopic surgery was conducted after two cycles of preoperative chemotherapy; the middle turbinate, ethmoid sinus, lamina papyracea, skull-base bone, and corresponding dura of the right side were resected intraoperatively. There was no local recurrence of the tumor after I year of follow-up.

Abbreviation: MRI, magnetic resonance imaging.

that is considered safe should be the soft tissue beyond the bony box (ie, the dura mater or periorbit). Our data showed that when the tumor involves the sphenoid sinus, dura mater, and intraorbital region, these locations are adjacent to important structures such as the internal carotid, optic nerve, eye, and brain; when tumors break outside the boundary of the paranasal sinus and involve the infratemporal fossa and pterygopalatine fossa, SAB is not available, so the SAP was difficult to obtain.

When the tumors were adjacent to important structures, which, if damaged, may lead to impaired functions postoperatively and make it difficult to perform a radical resection and obtain SAP, or if the patient's condition was unsuitable or the patient was reluctant to undergo an open approach, preoperative adjuvant therapy followed by MIER and postoperative adjuvant therapy were considered. According to the size of the tumor, in the preoperative adjuvant therapy group, 11 of the 17 cases faced the risk of postoperative optic nerve or internal carotid damage, and the others faced the risk of an inability to resect the tumor completely if preoperative therapies were not accepted. After preoperative adjuvant therapies, the patients could receive radical excision and obtain SAP. Finally, these patients could observe the preservation of the function of important structures. This group had a decreased 5-OS rate and shorter survival time than did the no-preoperative-therapy group but still had an improved outcome compared to the ACFR group (49 months for MIER vs 18 months for ACFR). The poorer survival in this group may be associated with the primary extension of the tumor. ${ }^{5}$ A meta-analysis showed that the higher survival rate of
MIER versus ACFR was related to tumor size and that orbital involvement was a significant prognostic factor. ${ }^{8-20}$ Despite allowing radical resection and preserving the functions of important structures, the curative effects of preoperative adjuvant therapy still require further investigation in studies with a sufficient sample size and prolonged follow-up.

Lund and $\mathrm{Wei}^{8}$ advocated that if the skull base bone is involved, the adjacent dura should be resected and that in cases of ONB or when the dura is affected, the olfactory bulbs and tracts could be removed, which is in accordance with our theory of resecting the SAB and obtaining a SAP in operation. Regrettably, there is a limitation in our study. Only six of the 15 cases involving the skull base bone underwent dura resection; the other nine cases that did not receive a dura excision have not had an SAP in our early experience, which might be associated with poor survival. Among these nine patients, eight obtained a negative margin, but four developed local recurrence after operation. This observation reflected the urgency of setting a widely acceptable operation standard.

In our cohort, the positive margin rate was equivalent to that in a previous report, and the recurrence rate (15\%) was lower than that of ACFR (22.1\%) ${ }^{16}$ indicating that MIER can achieve adequate resection without increasing the local recurrence in selected patients. The positive margin is the independent predictive factor when focusing on OS in our study and is related to decreased OS; this is consistent with the literature, ${ }^{16}$ but when focusing on DFS or recurrence, it is not. The probable reason is that the margin and SAP have collinearity. The positive-margin cases were all included in the 
Table 5 Summary of prognostic factors in multivariate analysis

\begin{tabular}{|c|c|c|}
\hline Prognostic factors & Hazard ratio $(95 \% \mathrm{Cl})$ & $P$-value \\
\hline \multicolumn{3}{|l|}{ Overall survival } \\
\hline Sex, female vs male & $1.63(0.63-4.24)$ & 0.32 \\
\hline Age, $<50$ years vs $\geq 50$ years & $4.32(1.64-11.37)$ & 0.01 \\
\hline T classification, $\mathrm{TI}-2$ vs T3-4 & $0.41(0.11-1.54)$ & 0.19 \\
\hline Cervical node metastasis, absent vs present & $18.53(3.24-105.97)$ & 0.01 \\
\hline Margin, negative vs positive & $3.48(1.13-10.78)$ & 0.03 \\
\hline SAP, present vs absent & $7.93(2.18-28.82)$ & 0.01 \\
\hline Histology & & 0.01 \\
\hline Melanoma vs olfactory neuroblastoma & $16.03(1.54-166.41)$ & 0.02 \\
\hline Melanoma vs squamous cell carcinoma & $11.97(1.32-108.71)$ & 0.03 \\
\hline Melanoma vs others & $4.84(0.52-44.85)$ & 0.17 \\
\hline Melanoma vs sarcoma & $1.27(0.10-16.10)$ & 0.86 \\
\hline Preoperative therapy, yes vs no & $2.19(0.63-7.65)$ & 0.22 \\
\hline Postoperative therapy & & 0.19 \\
\hline Surgery + chemotherapy vs surgery alone & $1.32(0.44-3.90)$ & 0.62 \\
\hline Surgery + radiotherapy vs surgery alone & $0.68(0.18-2.62)$ & 0.57 \\
\hline Surgery + chemoradiotherapy vs surgery alone & $0.29(0.08-1.05)$ & 0.06 \\
\hline \multicolumn{3}{|l|}{ Disease-free survival } \\
\hline Sex, female vs male & $1.27(0.54-3.00)$ & 0.59 \\
\hline Age, $<50$ years vs $\geq 50$ years & $2.59(1.64-6.02)$ & 0.03 \\
\hline T classification, TI-2 vs T3-4 & $0.79(0.23-2.69)$ & 0.71 \\
\hline Cervical node metastasis, absent vs present & $8.61(1.70-43.60)$ & 0.01 \\
\hline Margin, negative vs positive & $2.30(0.79-6.76)$ & 0.13 \\
\hline SAP, present vs absent & $7.71(2.32-25.63)$ & 0.01 \\
\hline Histology & & 0.04 \\
\hline Melanoma vs olfactory neuroblastoma & $12.86(1.33-124.21)$ & 0.03 \\
\hline Melanoma vs squamous cell carcinoma & $12.31(1.45-104.69)$ & 0.02 \\
\hline Melanoma vs others & $4.57(0.53-39.65)$ & 0.17 \\
\hline Melanoma vs sarcoma & $3.74(0.34-41.17)$ & 0.28 \\
\hline Preoperative therapy, yes vs no & $1.49(0.44-5.00)$ & 0.52 \\
\hline Postoperative therapy & & 0.56 \\
\hline Surgery + chemotherapy vs surgery alone & $1.09(0.40-2.99)$ & 0.87 \\
\hline Surgery + radiotherapy vs surgery alone & $0.78(0.21-2.91)$ & 0.71 \\
\hline Surgery + chemoradiotherapy vs surgery alone & $0.46(0.14-1.50)$ & 0.20 \\
\hline \multicolumn{3}{|l|}{ Recurrence } \\
\hline Sex, female vs male & $0.87(0.27-2.77)$ & 0.87 \\
\hline Age, $<50$ years vs $\geq 50$ years & $1.79(0.58-5.52)$ & 0.31 \\
\hline $\mathrm{T}$ classification, $\mathrm{TI}-2$ vs $\mathrm{T} 3-4$ & $1.41(0.26-7.66)$ & 0.69 \\
\hline Cervical node metastasis, absent vs present & $2.99(0.24-37.80)$ & 0.40 \\
\hline Margin, negative vs positive & $0.91(0.19-4.44)$ & 0.91 \\
\hline SAP, present vs absent & $7.12(1.43-35.56)$ & 0.01 \\
\hline Histology & & 0.38 \\
\hline Melanoma vs olfactory neuroblastoma & $3.54(0.30-42.17)$ & 0.32 \\
\hline Melanoma vs squamous cell carcinoma & $7.23(0.79-66.16)$ & 0.08 \\
\hline Melanoma vs others & $2.38(0.24-23.30)$ & 0.46 \\
\hline Melanoma vs sarcoma & $5.60(0.44-71.72)$ & 0.19 \\
\hline Preoperative therapy, yes vs no & $1.07(0.21-5.47)$ & 0.94 \\
\hline Postoperative therapy & & 0.86 \\
\hline Surgery + chemotherapy vs surgery alone & $0.76(0.18-3.24)$ & 0.71 \\
\hline Surgery + radiotherapy vs surgery alone & $1.14(0.20-6.55)$ & 0.88 \\
\hline Surgery + chemoradiotherapy vs surgery alone & $0.60(0.13-2.66)$ & 0.50 \\
\hline
\end{tabular}

Note: $P$-values were calculated with a Cox proportional-hazards model.

Abbreviations: $\mathrm{Cl}$, confidence interval; vs, versus; SAP, safety anatomic plane.

without-SAP group in our results. Conversely, the patients with SAP all had a clear margin. SAP may thus be the better indicator for oncology safety compared to the margin. The conception of SAP is first suggested by us here, and its role needs more studies for verification.
It is generally accepted that most sinonasal cancers are radiosensitive. Radical surgical resection followed by postoperative radiotherapy with or without chemotherapy can improve the local control rates. ${ }^{21}$ However, in our multivariate analysis, the group of surgery alone compared to 
the group with surgery followed by postoperative radiology, to the chemotherapy group, and to the concurrent chemoradiotherapy group exhibited no statistically significant differences in OS, DFS, and recurrence. The interpretation of the results of postoperative adjuvant therapy for sinonasal cancers is very complicated due to the high variety in histology, the biological behavior, and the site and extent of the tumor. Blanch et $\mathrm{al}^{22}$ reported no survival benefit with the addition of radiotherapy to surgery for early-stage disease, and there is limited evidence suggesting that radiotherapy or chemotherapy can prolong the survival of patients with melanoma. ${ }^{14}$ Related studies involve small series with mixed histologies and stages, as well as insufficient lengths of follow-up, ${ }^{8,23}$ which render evaluation of the role of individual treatments difficult.

\section{Conclusion}

This was a large-sample-size single-center study conducted in China. It gives weight to the notion that MIER is a more effective and safer procedure compared to ACFR in appropriately selected patients; a radical resection should resect both the tumor and $\mathrm{SAB}$, thereby obtaining a SAP. In order to preserve the function of important structures, sometimes, radical resection is difficult to achieve; for those cases, preoperative adjuvant therapy may be necessary. However, larger and long-term prospective studies with control groups are required in future research to further confirm this conclusion.

\section{Acknowledgments}

Dr Demin Han and Dr Pingdong Li undertook the surgery and made substantial contributions to conception and design, and acquisition of data. This work was supported by The Beijing Municipal Administration of Hospital Clinical Medicine Development of Special Funding (number XMLX201311), the Beijing Municipal Science \& Technology Commission (number Z141107002514003), and The Key Scientific Research Projects of the Guangxi Zhuang Autonomous Region Health and Family Planning Commission (number 2011116).

\section{Disclosure}

The authors report no conflicts of interest in this work.

\section{References}

1. Stammberger H, Anderhuber W, Walch C, Papaefthymiou G. Possibilities and limitations of endoscopic management of nasal and paranasal sinus malignancies. Acta Otorhinolaryngol Belg. 1999;53(3): 199-205.
2. Lund V, Stammberger H, Nicolai P, et al; European Rhinologic Society Advisory Board on Endoscopic Techniques in the Management of Nose, Paranasal Sinus and Skull Base Tumours. European position paper on endosccopic management of tumor of the nose, paranasal sinuses and skull base. Rhinol Suppl. 2010;22:1-143.

3. Husain Q, Patel SK, Soni RS, Patel AA, Liu JK, Eloy JA. Celebrating the golden anniversary of anterior skull base surgery: reflections on the past 50 years and its historical evolution. Laryngoscope. 2013;123(1): 64-72.

4. Snyderman CH, Carrau RL, Kassam AB, et al. Endoscopic skull base surgery: principles of endonasal oncological surgery. J Surg Oncol. 2008;97(8):658-664.

5. Bhayani MK, Yilmaz TA, Sweeney A, et al. Sinonasal adenocarcinoma: a 16-year experience at a single institution. Head Neck. 2014; 36(10):1490-1496.

6. Su SY, Kupferman ME, Demonte F, Levine NB, Raza SM, Hanna EY. Endoscopic resection of sinonasal cancers. Curr Oncol Rep. 2014; 16(2):369.

7. Harrow BR, Batra PS. Sinonasal quality of life outcomes after minimally invasive resection of sinonasal and skull-base tumors. Int Forum Allergy Rhinol. 2013;3(12):1013-1020.

8. Lund V, Wei W. Endoscopic surgery for malignant sinonasal tumours: an eighteen year experience. Rhinology. 2015;53(3):204-217.

9. Edge S, Byrd DR, Compton C, Fritz A, Greene F, Trotti A. AJCC Cancer Staging Handbook. New York, NY: Springer; 2010.

10. Patel S, Singh B, Polluri A, Bridger P, Cantu G, Cheesman A. Craniofacial surgery for malignant skull base tumors: report of an international collaborative study. Cancer. 2003;98(6):1179-1187.

11. Nicolai $P$, Castelnuovo P, Lombardi D, et al. Role of endoscopic surgery in the management of selected malignant epithelial neoplasms of the naso-ethmoidal complex. Head Neck. 2007;29(12):1075-1082.

12. Lund V, Howard D, Wei W. Endoscopic resection of malignant tumors of the nose and sinuses. Am J Rhinol. 2007;21(1):89-94.

13. Hanna E, Demonte F, Ibrahim S, Roberts D, Levine N, Kupferman M. Endoscopic resection of sinonasal cancers with and without craniotomy: oncologic results. Arch Otolaryngol Head Neck Surg. 2009;135(12): 1219-1224.

14. Haerle SK, Gullane PJ, Witterick IJ, Zweifel C, Gentili F. Sinonasal carcinomas: epidemiology, pathology, and management. Neurosurg Clin N Am. 2013;24(1):39-49.

15. Arnold A, Ziglinas $P$, Ochs K, et al. Therapy options and long-term results of sinonasal malignancies. Oral Oncol. 2012;48(10):1031-1037.

16. Komotar RJ, Starke RM, Raper DM, Anand VK, Schwartz TH. Endoscopic endonasal compared with anterior craniofacial and combined cranionasal resection of esthesioneuroblastomas. World Neurosurg. 2013; 80(1-2):148-159.

17. Suh JD, Ramakrishnan VR, Chi JJ, Palmer JN, Chiu AG. Outcomes and complications of endoscopic approaches for malignancies of the paranasal sinuses and anterior skull base. Ann Otol Rhinol Laryngol. 2013;122(1):54-59.

18. Wellman B, Traynelis V, McCulloch T, Funk G, Menezes A, Hoffman H. Midline anterior craniofacial approach for malignancy: results of en bloc versus piecemeal resections. Skull Base Surg. 1999;9(1):41-46.

19. Castelnuovo P, Dallan I, Battaglia P, Bignami M. Endoscopic endonasal skull base surgery: past, present and future. Eur Arch Otorhinolaryngol. 2010;267(5):649-663.

20. Devaiah A, Andreoli M. Treatment of esthesioneuroblastoma: a 16-year meta-analysis of 361 patients. Laryngoscope. 2009;119(7): $1412-1416$.

21. Won TB, Choi KY, Rhee CS, et al. Treatment outcomes of sinonasal malignant melanoma: a Korean multicenter study. Int Forum Allergy Rhinol. 2015;5(10):950-959.

22. Blanch J, Ruiz A, Alos L, Traserra-Coderch J, Bernal-Sprekelsen M. Treatment of 125 sinonasal tumors: prognostic factors, outcome, and follow-up. Otolaryngol Head Neck Surg. 2004;131(6):973-976.

23. Michel J, Fakhry N, Santini L, Mancini J, Giovanni A, Dessi P. Sinonasal adenoid cystic carcinomas: clinical outcomes and predictive factors. Int J Oral Maxillofac Surg. 2013;42(2):153-157. 


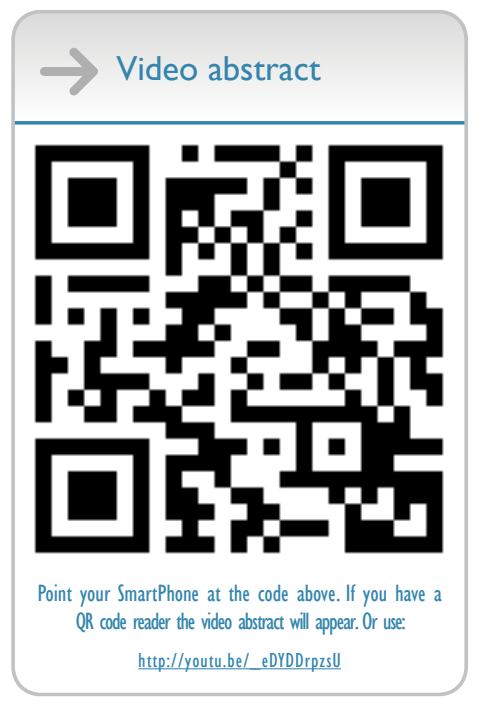

Therapeutics and Clinical Risk Management

\section{Publish your work in this journal}

Therapeutics and Clinical Risk Management is an international, peerreviewed journal of clinical therapeutics and risk management, focusing on concise rapid reporting of clinical studies in all therapeutic areas, outcomes, safety, and programs for the effective, safe, and sustained use of medicines. This journal is indexed on PubMed Central, CAS,
EMBase, Scopus and the Elsevier Bibliographic databases. The manuscript management system is completely online and includes a very quick and fair peer-review system, which is all easy to use. Visit $\mathrm{http}: / / \mathrm{www}$.dovepress.com/testimonials.php to read real quotes from published authors.

Submit your manuscript here: http://www.dovepress.com/therapeutics-and-clinical-risk-management-journal 